\title{
The complete plastid genome of Scopolia parviflora (Dunn.) Nakai (Solanaceae)
}

\author{
Jin Hee Park ${ }^{1}$ and Jungho Lee ${ }^{2 *}$ \\ ${ }^{1}$ Freshwater Bioresources Research Division, Nakdonggang National Institute of Biological Resources, Sangju 742-350, Korea \\ ${ }^{2}$ Green Plant Institute, B-301, Heungdeok IT Valley, Giheung-gu, Yongin 446-908, Korea \\ (Received 7 March 2016; Revised 17 March 2016; Accepted 18 March 2016)
}

\begin{abstract}
Scopolia parviflora of the family Solanaceae is an endemic species of Korea and a traditional Korean medicinal plant. The plastid genome was sequenced by next-generation sequencing (NGS) method. The characterized cp genome is $156,193 \mathrm{bp}$ in size; the large single-copy (LSC) region is $86,364 \mathrm{bp}$, the inverted repeat (IR) is $25,905 \mathrm{bp}$, and the small single copy (SSC) region is $18,019 \mathrm{bp}$. The overall GC content of the plastid genome amounts to $37.61 \%$. The cp genome contains 113 genes and 21 introns, including 80 proteincoding genes, four RNA genes, 30 tRNA genes, 20 group II introns, and one group I intron. A phylogenetic analysis showed that Scopolia parviflora was closely related to Hyoscyamus niger.
\end{abstract}

Keywords: Chloroplast, Scopolia parviflora, genome sequence, medicinal plant

Michikwangipul, Scopolia parviflora (Dunn.) Nakai (Dunn, 1912; Nakai, 1933) of the family Solanaceae, limitedly occurs in Korean peninsula, and is similar to $S$. japonica (Maximowicz, 1873) occurring in Japan. S. parviflora is distinguished from $S$. japonica by ITS and some phenetic characters (Hong and Paik, 2001; Kim et al., 2003). The members of the genus Scopolia are known as medicinal plants (Mino, 2002; Jung et al., 2003; Min et al., 2007). The genetic makeup of the plastid genome of Scopolia is poorly known. Here we sequenced the chloroplast genome of Scopolia parviflora as a representative of the genus Scopolia.

\section{Materials and Methods}

The plant material of Michikwangipul used in this study was collected from the wild population of Mt. Cheonma, Korea

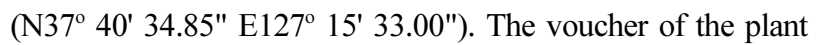
specimen (Parkjh 20150505-141) was deposited in NNIBR, Herbarium in Nakdonggang National Institute of Biological Resources. The total DNA was prepared as described by Lee et al. (2015). The Illumina paired-end (PE) genomic library of 200 bp was constructed and sequenced using an Illumina HiSeq
2000 platform. The plastid sequence was obtained using CLC Genomics Workbench version 7.05 as described by Jeong et al. (2014). Circular structures of each replicon were confirmed by polymerase chain reaction (PCR) amplification at their ends and by joining of Sanger sequence reads derived from the amplicons. The assemblies were further verified by examining paired-end distance and depth after re-mapping reads on the contig sequences. The BLAST searches of a large contig were verified to be plastid genomes. For gene annotation of organelle genomes, protein-coding and ribosomal RNA genes were annotated using DOGMA (http://dogma.ccbb.utexas.edu/; Wyman et al., 2004). The boundaries of each annotated gene were manually determined by comparison with orthologous genes from other known cp genomes. Genes encoding tRNAs were first predicted using tRNAscan (http://lowelab.ucsc.edu/ tRNA scan-SE; Lowe and Eddy, 1997) and ARAGORN version 1.2 (http://130.235.46.10/ARAGORN/; Laslett and Canback, 2004), and were manually verified by predicting the tRNA secondary structure. Circular genome maps were drawn using GenomeVx (Conant and Wolfe, 2008) followed by manual modification. The sequencing data and gene annotations were submitted to GenBank with accession number

\footnotetext{
*Author for correspondence: jlee@greenplant.re.kr
} 
KU900232. DNA sequences of seven $\mathrm{cp}$ protein genes, including $p s a A, p s a B, p s b A, p s b B, p s b C, p s b D$, and $r b c L$ were used to construct $\mathrm{cp}$ phylogenetic tree by Maximum Parsimony criterion using Paup ver. 6.0. Bootstrap and jackknife analyses of the MP tree were also performed with 1,000 replicates.

\section{Results and Discussion}

The cp-genome of Scopolia parviflora, was determined (Fig. 1) and found to be $156,193 \mathrm{bp}$ in length. It includes small and large single copy (SSC, LSC) regions of $18,019 \mathrm{bp}$ and 86,364 bp, respectively, separated by a pair of $25,905 \mathrm{bp}$ inverted repeats (IRs). A total of 113 genes were detected, including 80 protein coding genes, 30 tRNA genes, and four rRNA genes (Table 1). This cp-genome was also found to contain 21 different introns, including 20 group II introns and a group I intron with a cyanobacterial origin (Besendahl et al., 2000) found within the trnL_ua gene. Three protein coding genes, including $c l p P$, rps12, and $y c f 3$, contain two group II introns (clpP.i1, clpP.i2, rps12.i1, rps12.i2, $y c f 3 . i 1$ and $y c f 3 . i 2)$, and 14 genes contain a

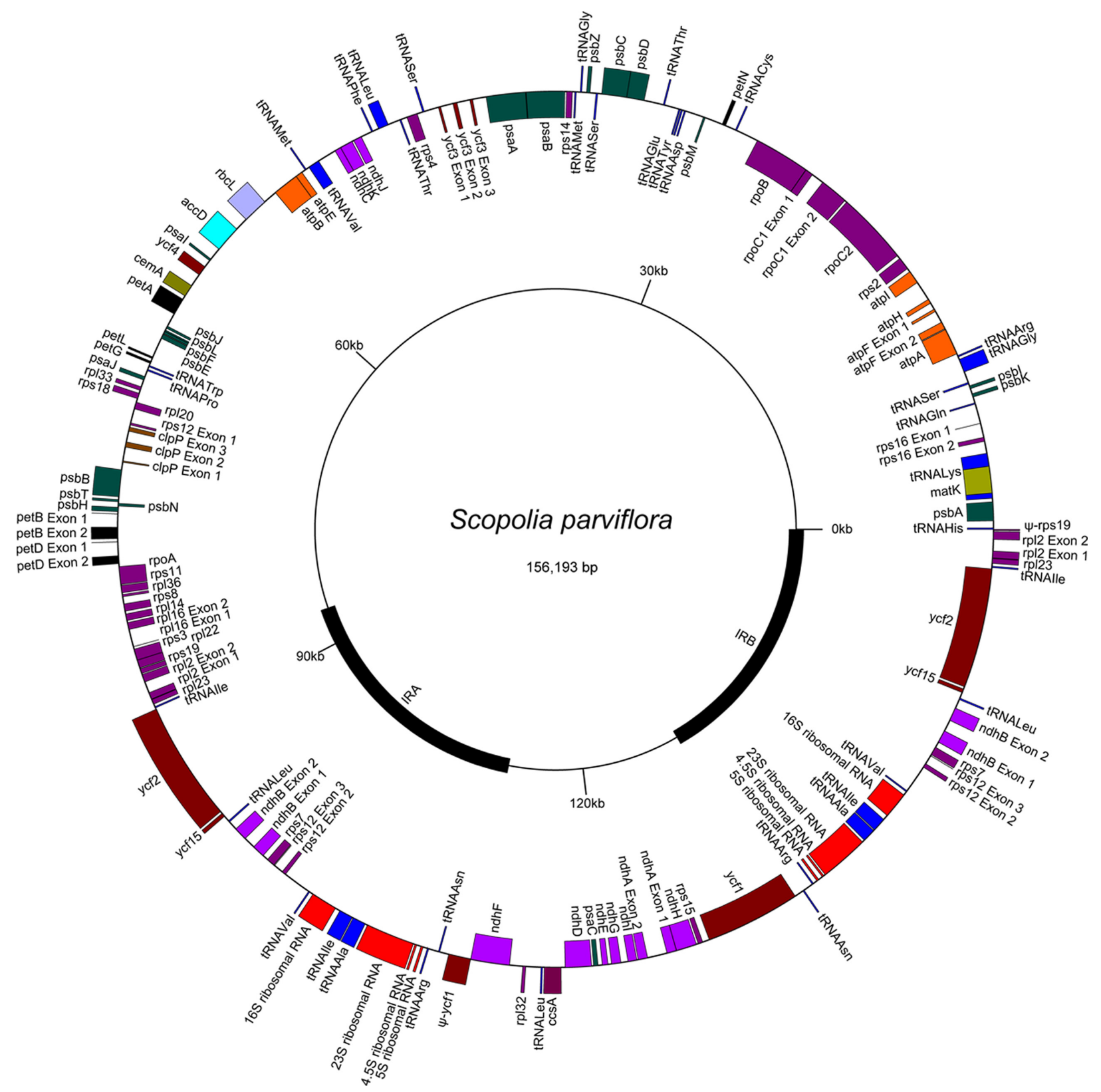

Fig. 1. Plastid genome map of Scopolia parviflora. 


\section{Gene}

Genetic system genes

Conserved off

Maturase $\mathrm{K}$

RNA polymerase

Ribosomal protein

Large subunits

Small subunits

Photosynthesis genes

\begin{abstract}
Acetyl-CoA carboxylase
\end{abstract}
ATP-dependent Clp protease

ATP synthase

Cytochrome b

Cytochrome b/f

Cytochrome $\mathrm{f}$

Cytochrome $\mathrm{C}$ biogenesis

Membrane protein

NADH dehydrogenase

Photosystem I

Photosystem II

Rubisco

\begin{tabular}{|c|c|c|c|c|c|c|c|c|c|}
\hline Ribosomal RNA & $r r n 16 S \times 2$ & $r r n 23 S \times 2$ & $r r n 4.5 S \times 2$ & $r r n 5 S \times 2$ & & & & & \\
\hline \multirow[t]{4}{*}{ Transfer RNA } & trnA_UGC $C^{*} \times 2$ & $\operatorname{trn} C \_G C A$ & trnD_GUC & $\operatorname{trnE} E_{-} U U C$ & $\operatorname{trn} F \_G A A$ & $\operatorname{trnf} M \_C A U$ & $\operatorname{trn} G_{-} G C C$ & $\operatorname{trn} G_{-} U C C^{*}$ & trnH_GUG \\
\hline & $\operatorname{trn} I_{-} C A U \times 2$ & trnI_GAU ${ }^{*} \times 2$ & $\operatorname{trnK} K_{-} U U U^{*}$ & $t r n L \_C A A \times 2$ & $t r n L \_U A A^{*}$ & trnL_UAG & trnM_CAU & $t r n N \_G U U \times 2$ & $t r n P \_U G G$ \\
\hline & $\operatorname{trn} Q \_U U G$ & $t r n R \_A C G \times 2$ & $t r n R \_U C U$ & trnS_GCU & trnS_GGA & $t r n S \_U G A$ & $t r n T \_G G U$ & $t r n T \_U G U$ & $t r n V_{-} G A C \times 2$ \\
\hline & $\operatorname{trn} V_{-} U A C^{*}$ & $\operatorname{trn} W \_C C A$ & $t r n Y \_G U A$ & & & & & & \\
\hline
\end{tabular}

$y c f 1$

$y c f 2 \times 2$

$y c f 3 * *$

$y c f 4$

$y c f 15$

rров

$r p o C 1^{*}$

rpoC2

$r p l 2 * 2 \quad r p l 14 \quad$ rpll6*

rps 2

rps $16^{*}$

rps 3

$\operatorname{rps} 18$

rps 4

rps19

$a c c D$

clpP**

atpA

petB*

petD*

petA

$\operatorname{ccs} A$

cemA

$n d h A^{*}$

$n d h J$

psaA

$p s b A$

psbH

$n d h B^{*} \times 2$

$n d h K$

psaB

$p s a B$
$p s b B$

psbI

atpE

$a t p F^{*}$

$a t p H$

petL $\quad \operatorname{pet} N$

$a t p I$

*intron ${ }^{\alpha} \mathrm{cp}$ genome contains a copy of $r p s 12$ exon 1 in LSC and two copies of $r p s 12$ exon 2 and 3 in IR 


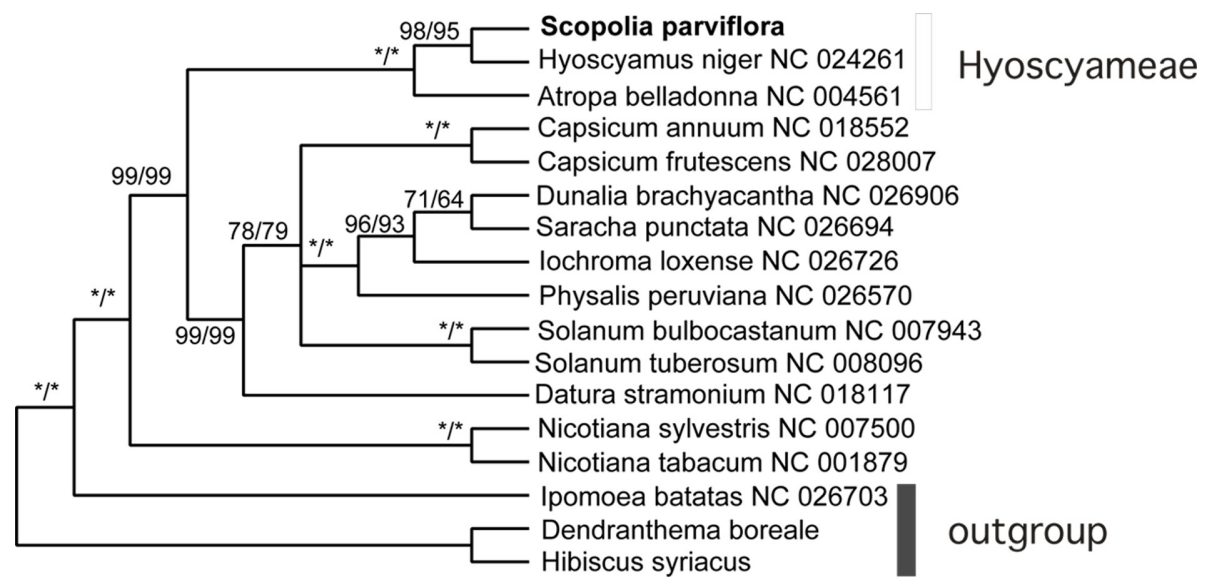

Fig. 2. Maximum parsimonious tree of 14 Solanaceae plastids, using seven protein coding gene ( $p s a A, p s a B$, $p s b A, p s b B$, $p s b C$, $p s b D$, and $r b c L$ ) sequences. */*: Bootstrap value $100 \% / J a c k n i f e$ value $100 \%$.

single group II intron: rpoC1.i, rpl2.i, rpl16.i, rps16.i, atpF.i, petB.i, petD.i,ndhA.i, ndhB.i, trnA_ugc.i, trnG_ucc.i, trnI_gau.i, trnK_uuu.i, and trnV_uac.i. Among the 20 group II introns, the intron in rps 12, between exons 1 and 2, is trans-splicing, while the other 19 group II introns are cis-splicing.

Seventeen genes, five introns, and parts of three genes and an intron are found within the IR, which has two copies. These 17 genes include six protein-coding genes ( $n d h B, r p l 2, r p l 23$, $r p s 7, y c f 2, y c f 15)$, all four rRNA genes (16S, 23S, 4.5S, 5S), and seven tRNA genes (trnA_ugc, trnI_cau, trnI_gau, trnL_caa, trnN_guu, trnR_acg, trnV_gac). The five introns are ndhB.i, rpl2.i, trnA_ugc.i, trnI_gau.i, and rps12.i2. The IR contains the 5' end of $y c f 1$ at the border with the SSC, resulting in one intact $y c f 1$ and a 1,473-bp $\psi-y c f 1$ in the cp-genome. The IR also contains the 5' end of rps19 at the border with the LSC, resulting in one intact rps 19 and a 84-bp $\psi$-rps 19 in the cp-genome. In addition, the IR contains parts of the rps 12 gene. This rps12 gene consists of three exons, rps12.e1, rps12.e2, and rps12.e3, rps12.e1 is in the LSC, but rps12.e2 and rps12.e3 are in the IR. Thus, the genome contains a single copy of rps12.e1 but has two copies of rps12.e2 and rps12.e3. A cis-splicing group II intron, rps 12.i2, intervenes between rps12.e2 and rps12.e3, but a trans-splicing intron, rps12.i1t, occurs between rps12.e1 and rps12.e2. The rps12.i1t is split into two pieces, rps12.i1t1 and rps12.i1t2, because the rps12 gene is transcribed in two separate operons, the $c l p P$ operon (clpP-rps12.e1-rps12.i1t1-rpl20) and the 3' rps12 operon (rps12.i1t2-rps12.e2-rps12.i2-rps12.e3-rps7-ndhB).

Currently, more than 20 plastid genomes have been deposited in Genbank from 10 genera of Solanaceae. Phylogenetic analysis showed that Scopolia parviflora formed a strong clade with Hyoscyamus niger (Sanchez-Puerta \& Abbona, 2014) and Atropha belladonna (Schmitz-Linneweber et al., 2002), and that Hyoscyamus niger was the closest to Scopolia parviflora. The results support monophyly of the tribe Hyoscyameae of Solanaceae. The three plants are known to be highly toxic and are also used as medicine. In contrast, another toxic plant, Datura (Yang et al., 2014), claded with potato (Fig. 2).

In Korea, two species of the genus Scopolia have been documented. One is purple flowered Scopolia parviflora (Dunn.) Nakai (1933) and the other is yellow flowered $S$. lutescens Y. Lee (1993). In contrast, purple flowered Scopolia japonica Maxim. (1873) and yellow flowered Scopolia japonica Maxim. f. lutescens Sugim. (1977) also occur in Japan. Currently, ITS sequence analysis suggested that Scopolia parviflora and S. japonica were clearly distinguished, but that Scopolia parviflora and S. lutescens Y. Lee were indistinguishable (Kim et al., 2003). Plastid DNA sequence of Scopolia japonica from Japanese collection (voucher Tsugaru $\&$ Sawada, 17731) was only available in $n d h F$ (Genbank EU580945). Without 162 ambiguous sequence of EU580945, Scopolia japonica had 5 SNPs in $n d h F$ distinguished from Scopolia parviflora. Further study of comparative plastid genomics would help our understanding on the relationship among the Scopolia species.

\section{Acknowledgments}

This work was supported by a grant from the National Institute of Biological Resources under the Ministry of Environment, Republic of Korea.

\section{Literature Cited}

Besendahl, A., Y. L. Qiu, Lee, J. D. Palmer and D. Bhattacharya. 2000. The cyanobacterial origin and vertical transmission of 
the plastid tRNA(Leu) group-I intron. Current Genetics 37: $12-23$.

Conant, G. C. and K. H. Wolfe. 2008. GenomeVx: simple webbased creation of editable circular chromosome maps. Bioinformatics 24: 861-862.

Dunn, S. T. 1912. Some additions to Korean flora. Bulletin of Miscellaneous Information, Royal Gardens, Kew 1912: 108-109.

Hong, S.-P. and J.-H. Paik. 2001. Leaf epidermal microstructure of the genus Scopolia Jacq. s.l. (Solanaceae-Hyoscymeae) and its systematic significance. Korean Journal of Plant Taxonomy 31: 267-282. (in Korean)

Jeong, H., J. M. Lim, J. Park, Y. M. Sim, H. G. Choi, J. Lee and W. J. Jeong. 2014. Plastid and mitochondrion genomic sequences from Arctic Chlorella sp. ArM0029B. BMC Genomics 15: 286. doi: 10.1186/1471-2164-15-286

Jung, H. Y., S. M. Kang, Y. M. Kang, M. J. Kang, D. J. Yun, J. D. Bahk, J. K. Yang and M. S. Choi. 2003. Enhanced production of scopolamine by bacterial elicitors in adventitious hairy root cultures of Scopolia parviflora. Enzyme and Microbial Technology 33: 987-90.

Kim, Y.-D., J.-H. Paik, S.-H. Kim and S.-P. Hong. 2003. Phylogeny of Scopolia Jaq. s. str. based on ITS sequences. Korean Journal of Plant Taxonomy 33: 373-386. (in Korean)

Laslett, D. and B. Canback. 2004. ARAGORN, a program to detect tRNA genes and tmRNA genes in nucleotide sequences. Nucleic Acids Research 32: 11-16. doi: 10.1093/ nar/gkh152

Lee, M., J. Park, H. Lee, S.-H. Sohn and J. Lee. 2015. Complete chloroplast genomic sequence of Citrus platymamma determined by combined analysis of Sanger and NGS data. Horticulture and Environmental Biotechnology 56: 704-711.

Lee, Y. 1993. New taxa on Korean flora (5). Korean Journal of Plant Taxonomy 23: 263-268 (in Korean).

Lowe, T. M. and S. R. Eddy. 1997. tRNAscan-SE: a program for improved detection of transfer RNA genes in genomic sequence. Nucleic Acids Research 25: 955-964.

Maximowicz, C. J. 1873. Diagnoses plantarum novarum Japoniae et Mandshuriae. Bulletin de l'Acadeìmie Impeìriale des Sciences de St. Peìtersbourg 18: 35-72.

Min, J. Y., H. Y. Jung, S. M. Kang, Y. D. Kim, Y. M. Kang, D. J. Park, D. T. Prasad and M. S. Choi. 2007. Production of tropane alkaloids by small-scale bubble column bioreactor cultures of Scopolia parviflora adventitious roots. Bioresource Technology 98: 1748-1753.

Mino, Y. 2002. Amino acid sequences of ferredoxins from Scopolia japonica and Lycium chinense: their similarities to that of Datura arborea. Biological Pharmceutical Bulletin 25: 13671369.

Nakai, T. 1933. Notulae ad Plantas Japoniae \& Koreae XLIII. Botanical Magazine 47: 235-267.

Sanchez-Puerta, M. V. and C. C. Abbona. 2014. The chloroplast genome of Hyoscyamus niger and a phylogenetic study of the tribe Hyoscyameae (Solanaceae). PLoS One 9: e98353. doi:10.1371/journal.pone.0098353.

Schmitz-Linneweber, C., R. Regel, T. G. Du, H. Hupfer, R. G. Herrmann and R. M. Maier. 2002. The plastid chromosome of Atropa belladonna and its comparison with that of Nicotiana tabacum: the role of RNA editing in generating divergence in the process of plant speciation. Molecular Biology and Evolution 19: 1602-1612.

Sugimoto, J. 1977. Notes on flora of Japan (3). Journal of Geobotany 26: 59-64. (in Japanese)

Wyman, S. K., R. K. Jansen and J. L. Boore. 2004. Automatic annotation of organellar genomes with DOGMA. Bioinformatics 20: 3252-3255. doi: 10.1093/bioinformatics/bth352

Yang, Y., Y. Dang, Q. Li, J. Lu, X. Li and Y. Wang. 2014. Complete chloroplast genome sequence of poisonous and medicinal plant Datura stramonium: organizations and implications for genetic engineering. PLoS One 9: e110656. doi: 10.1371/journal.pone.0110656 